\title{
Fidgetin-Like 2 siRNA Enhances the Wound Healing Capability of a Surfactant Polymer Dressing
}

\author{
Brian P. O’Rourke, ${ }^{1, \dagger}$ Adam H. Kramer, ${ }^{2, \dagger}$ Longyue L. Cao, ${ }^{3,4}$ \\ Mohammed Inayathullah, ${ }^{5}$ Hillary Guzik, ${ }^{6}$ Jayakumar Rajadas, ${ }^{5}$ \\ Joshua D. Nosanchuk, ${ }^{7,8}$ and David J. Sharp ${ }^{1,2,9,10, *}$ \\ ${ }^{7}$ MicroCures, Inc., Research and Development, Bronx, New York \\ ${ }^{2}$ Physiology and Biophysics, and ${ }^{6}$ Analytical Imaging Facility, Albert Einstein College of Medicine, Bronx, New York \\ ${ }^{3}$ Department of Medicine, Children's Hospital Boston, Boston, Massachusetts. \\ ${ }^{5}$ Biomaterials and Advanced Drug Delivery Laboratory, Stanford University, Palo Alto, California. \\ Departments of ${ }^{4}$ Developmental and Molecular Biology, ${ }^{7}$ Medicine, ${ }^{8}$ Microbiology and Immunology, ${ }^{9}$ Ophthalmology \\ and Visual Sciences, and ${ }^{10}$ Neuroscience, Albert Einstein College of Medicine, Bronx, New York. \\ ${ }^{\dagger}$ Co-first authors.
}

Microtubules (MTs) are intracellular polymers that provide structure to the cell, serve as railways for intracellular transport, and regulate many cellular activities, including cell migration. The dynamicity and function of the MT cytoskeleton are determined in large part by its regulatory proteins, including the recently discovered MT severing enzyme Fidgetin-like 2 (FL2). Downregulation of FL2 expression with small interfering RNA (siRNA) results in a more than twofold increase in cell migration rate in vitro as well as translates into improved wound-healing outcomes in in vivo mouse models. Here we utilized a commercially available surfactant polymer dressing (SPD) as a vehicle to deliver FL2 siRNA. To this end we incorporated collagen microparticles containing FL2 siRNA into SPD (SPD-FL2-siRNA) for direct application to the injury site. Topical application of SPD-FL2 siRNA to murine models of full-thickness excision wounds and full-thickness burn wounds resulted in significant improvements in the rate and quality of wound healing, as measured clinically and histologically, compared with controls. Wound healing occurred more rapidly and with high fidelity, resulting in properly organized collagen substructure. Taken together, these findings indicate that the incorporation of FL2 siRNA into existing treatment options is a promising avenue to improve wound outcomes.

Keywords: Fidgetin-like 2, siRNA, wound healing, re-epithelialization, regeneration, microtubules

\section{INTRODUCTION}

WOUND HEALING Is a complex physiological process that, when not effectively completed, can lead to serious complications such as chronic wounds, infections, and ultimately amputations. ${ }^{1}$ Current approaches in acute wound healing are generally limited to passive dressings, skinlike matrices, and growth factorbased approaches that address only the initial, inflammatory phase of the healing process. ${ }^{2-5}$ One area undergoing significant development is the use of poloxamer-based hydrogels called surfactant polymer dressings

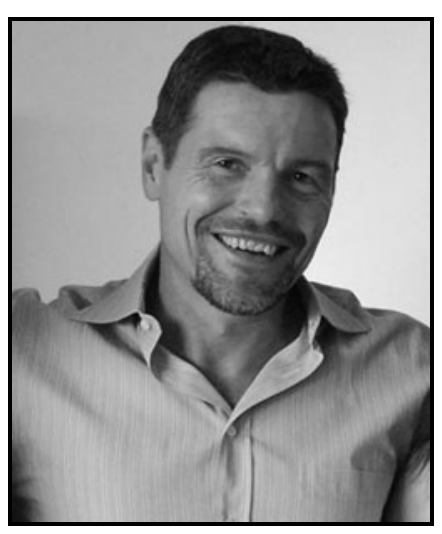

David J. Sharp, PhD

Submitted for publication August 1, 2018 Accepted in revised form September 7, 2018.

${ }^{*}$ Correspondence: Physiology and Biophysics, Albert Einstein College of Medicine, 1300 Morris Park Avenue, Ullmann Building Room 223, Bronx, NY 10461

MicroCures, Inc., 1300 Morris Park Ave., Forchheimer Building Suite 726, Bronx, NY 10461. (e-mail: david.sharp@einstein.yu.edu; David@ MicroCures.com)

(c) Brian O'Rourke et al., 2018; Published by Mary Ann Liebert, Inc. This Open Access article is distibuted under the terms of the Creative Commons Attribution Noncommercial License (http:// creativecommons.org/licenses/by-nc/4.0), which permits any noncommercial use, distibution, and reproduction in any medium, provided the original authors and the source are cited. 
(SPDs). ${ }^{6-8}$ These physiologically amenable polymers can repair damaged cell membranes, effectively encapsulate small interfering RNA (siRNA), and increase hydration of the wound.$^{9-11}$ Furthermore, these SPDs are clinically useful as they remain at the site of application and restrict drug delivery to the desired areas. Identification and incorporation of a therapeutically useful siRNA target into an SPD could be used to further improve wound outcomes.

Our previous work that involved screening of biological agents for cell migration phenotypes identified a novel regulator of the microtubule (MT) cytoskeleton, termed Fidgetin-like 2 (FL2), as an important negative regulator of cell migration. ${ }^{12}$ siRNA-mediated depletion of FL2 results in a significant increase in the velocity and directionality of keratinocyte and fibroblast motility in vitro. ${ }^{12}$ Moreover, we found that in vivo depletion of FL2 strongly promoted wound closure and repair. Of note, FL2 depletion enhanced healing by inducing a targeted, transient disruption of cortical MT within the leading edge of fibroblasts and keratinocytes. ${ }^{12}$ This localized alteration bypasses the manipulation of larger signaling cascades, which have been shown to induce numerous unintended side effects. ${ }^{3,5,13}$ Having identified FL2 as a promising therapeutic target we began development of an SPD containing FL2 siRNA.

As poloxamer technology has matured, commercially available SPDs are more widely used. We identified one biocompatible and nonionic SPD containing poloxamer 188 as a candidate for siRNA incorporation. ${ }^{14}$ This SPD has previously been shown to improve wound-healing outcomes (improved wound closure rates, reduction of inflammation, pain, and odor) in nonhealing wounds and is now in a U.S.-based clinical trial to determine its efficacy in the treatment of wounds of mixed etiologies. ${ }^{15,16}$ Looking to utilize the complimentary healing mechanisms of FL2 siRNA and SPD, we synthesized collagen microparticles containing FL2 siRNA and incorporated these into the SPD for topical application in two in vivo wound-healing models.

Here, we demonstrate that the incorporation of FL2 siRNA collagen microparticles into SPD (SPDFL2-siRNA) significantly enhances healing outcomes compared with controls (SPD without microparticles and SPD-Control-siRNA), as measured clinically and histologically in two murine wound-healing models, namely full-thickness excision and fullthickness burn. Following SPD-FL2-siRNA treatment, there was a decrease in inflammation and an increase in revascularization at the wound site, while cell proliferation was unaffected. Wound healing occurred more rapidly and with high fidelity, resulting in properly organized collagen substructure. Taken together, these findings indicate that the incorporation of FL2 siRNA into existing treatment options is a promising avenue to improve wound management.

\section{CLINICAL PROBLEM ADDRESSED}

Slow healing and open wounds are a serious medical problem faced by patients worldwide. Without proper re-epithelialization, these wounds are at risk of infection, increasing the risk of amputation or mortality. Slow-healing wounds also bring the risk of scarring and skin deformation. ${ }^{17} \mathrm{~A}$ therapeutic that can more quickly close wounds would directly address these issues.

\section{MATERIALS AND METHODS}

\section{Surfactant polymer dressing}

A nonionic SPD (PluroGel ${ }^{\circledR}$; Medline Industries, Inc.) was used for siRNA delivery.

\section{siRNA}

Sequences targeting mouse FL2 messenger RNA (mRNA) (SASI_Mm02_00354635; Sigma-Aldrich) or a nontargeting negative control siRNA (SIC001; Sigma-Aldrich) were used.

\section{Collagen microparticle and SPD incorporation}

Ten grams of sodium bis(2-ethylhexyl) sulfosuccinate (AOT; Sigma-Aldrich) was dissolved in $34 \mathrm{~mL}$-hexane, and $2 \mathrm{~mL} \mathrm{5 \%}$ collagen-I dissolved in acetic acid was added. The resulting microemulsion was stirred for $45 \mathrm{~min}$ until it became clear. This solution was then evaporated to remove hexane. The residue was washed and then suspended in nuclease-free water and lyophilized. Then $100 \mathrm{mg}$ of lyophilized powder was then treated with $1,000 \mu \mathrm{L}$ of $25 \mu \mathrm{M}$ siRNA solution and relyophilized. This material was then suspended in $1.25 \mathrm{~mL} \mathrm{SPD}$, at $4^{\circ} \mathrm{C}$ for $2 \mathrm{~h}$, and then lyophilized. The lyophilized powder was then added with $1.25 \mathrm{~mL}$ nuclease-free water and $1.25 \mathrm{~mL}$ SPD.

\section{In vivo studies}

Animal experiments were performed according to the guidelines published by the Institute of Laboratory Animal Resources of National Research Council, and animal care for this study was approved by the Institutional Animal Care and Use Committee of the Albert Einstein College of Medicine.

\section{Punch biopsy excision}

Before procedures, mice were anesthetized with a ketamine-xylazine cocktail. Female Balb/c mice 
(6-8 weeks; National Cancer Institute, Frederick, MD) were shaved and then wounded uniformly on their dorsa using a $5 \mathrm{~mm}$ punch biopsy tool. ${ }^{12}$ To counteract wound contraction typically seen in mice, we used an established silicone splinting method to encourage the formation of granulation tissue and re-epithelialization. ${ }^{18}$ Splints were checked multiple times a day during the study and replaced as needed. Wounds were treated with $10 \mu \mathrm{L}$ of SPD, SPD-Control-siRNA, or SPD-FL2-siRNA on days 0 , 2 , and 4.

\section{Burn protocol}

Before procedures, mice were anesthetized with a ketamine-xylazine cocktail. Female Balb/c mice (6-8 weeks; National Cancer Institute, Frederick, MD) were shaved and then burned uniformly on their dorsa using a $5 \mathrm{~mm}$ brass probe heated to $100^{\circ} \mathrm{C}{ }^{12,19}$ Mice were provided buprenorphine as needed following injury. Wounds were treated with $10 \mu \mathrm{L}$ of SPD, SPD-Control-siRNA, or SPD-FL2siRNA on days 0,2 , and 4 .

\section{Wound measurements}

Wounds were measured by investigators blinded to the treatment groups using surgical calipers. Measurements were made in four planes (N-S, WE, NE-SW, NW-SE), tabulated, and compared with day $0 .{ }^{18}$ Histologically, wound edges were defined by the distance between the first hair follicle encountered at each end of the wound.

\section{Histochemistry}

Wound sites were harvested, fixed overnight in HistoChoice, bisected through the center of the wound, and embedded in paraffin blocks. Sevenmicrometer sections were cut and slides were deparaffinized, washed in phosphate buffered saline (PBS)-0.01\% TritonX100, unmasked with antigen retrieval buffer tris(hydroxymethyl)aminomethane/ ethylenediaminetetraacetic acid $\mathrm{pH} \mathrm{9.0,} \mathrm{and} \mathrm{blocked}$ with 5\% normal goat serum in PBS. Sections were stained overnight with their respective antibody and visualized using a 3,3'-diaminobenzidine (DAB) kit (Vector Labs).

\section{Microscopy}

Whole wound sections were imaged with a P250 High Capacity Slide Scanner (3D Histech) using brightfield with a $20 \times$ air objective, numerical aperture 0.8 , and complementary metaloxide semiconductor (CMOS) color camera (VCCFC60FR19CL; CIS Corp.). Measurements of wound sizes were made using the CaseViewer analysis software (3D Histech). The size of wounds was determined by measuring the maximum length between wound edges. Quantifications of proliferating cell nuclear antigen (PCNA), cluster of differentiation 45 (CD45), and platelet endothelial cell adhesion molecule-1 (PECAM1) were conducted as described elsewhere. ${ }^{20,21}$ Herovici analysis was conducted following a previously described approach. ${ }^{22,23}$

\section{Statistical analysis}

Data are expressed as mean \pm standard error of the mean. Differences between treatment groups were calculated using unpaired Student's, MannWhitney nonparametric $t$-tests, or two-way analysis of variances. Significance was set at $p<0.05$.

\section{Commercial antibodies and stains}

Commercially produced antibodies used in immunohistochemistry included PECAM-1 (1:50; ab28364; Abcam), CD45 (1:200; ab10558; Abcam), PCNA (1:1,000; ab152112; Abcam), and Keratin 14 (1:500; 906004; BioLegend). Alternatively, sections were stained with hematoxylin (\#3530-32; Ricca Chemical Company) and eosin (\#17372-87-1; Acros Organics) or Herovici (\#KTHER; American MasterTech) following the manufacturer's suggested protocol.

\section{Quantitative PCR}

Skin of SPD, SPD-Control-siRNA, and SPD-FL2siRNA-treated mice was excised at set time points after wounding. The Next Advance Bullet Blender was used to pulverize the tissue, and RNA was extracted with Trizol reagent (15596-026; Invitrogen). Complementary DNA was synthesized the same day using the SuperScript IV First-strand synthesis system (18091050; Invitrogen). Quantitative PCR (qPCR) was performed using Life Technologies Universal Master Mix II (4440040) with FIGNL2 primers (Mm.PT.58.21940655; IDT Technologies). ActB (actin) served as the reference gene (Mm.PT.58.33540333; IDT Technologies). Resulting data were quantified using the comparative $2^{-\Delta \Delta \mathrm{Ct}}$ method. The average of control skin wounds was normalized to 1 and used for relative quantification.

\section{RESULTS}

\section{SPD-FL2-siRNA knocks down FL2 messenger RNA abundance}

Delivery of FL2-targeting siRNA to the cells within the wound zone was achieved by direct topical application of our therapeutic surfactant polymer containing siRNA microparticles (Fig. 1A). Reduction of FL2 levels was confirmed by qPCR analysis on injured skin 3 days post initial treatment (Fig. 1B). Wounds that received FL2 siRNA had a 51\% reduction in FL2 mRNA levels com- 


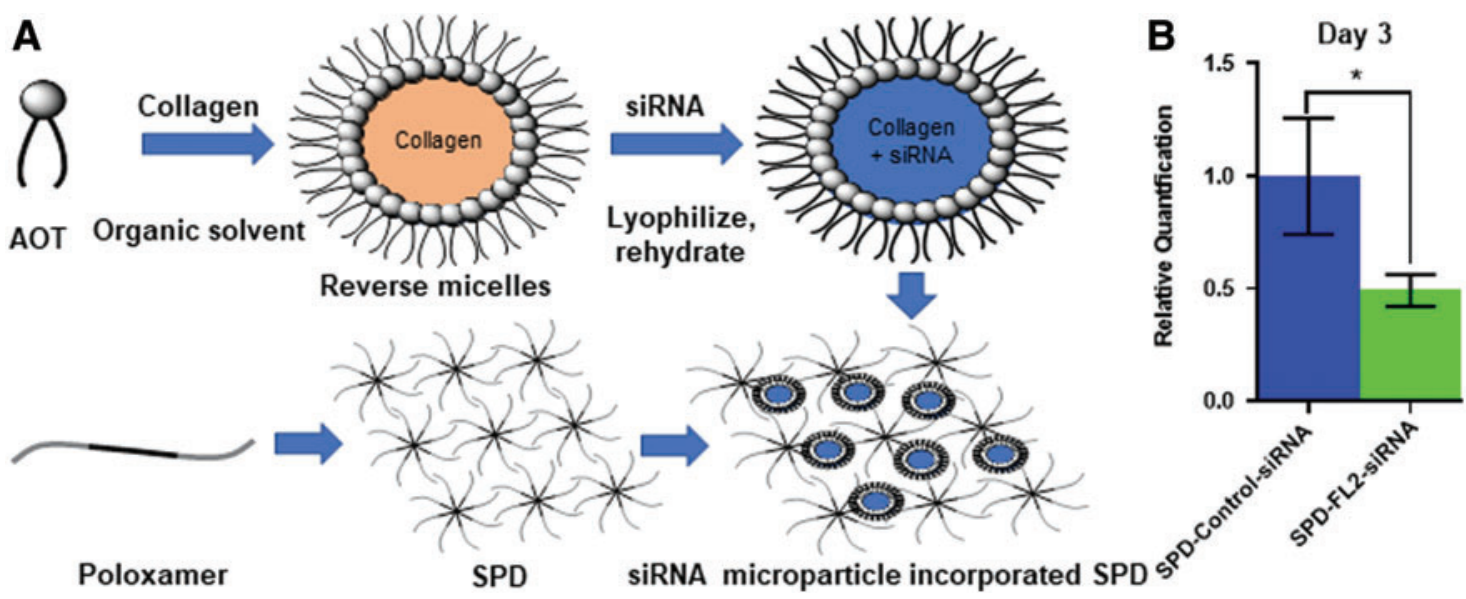

Figure 1. FL2 levels are reduced in injured skin treated with SPD-FL2-siRNA. (A) Schematic of microparticle siRNA incorporation into SPD. (B) Mice were burned and then treated at days 0 and 2 with the designated agent. Burns were harvested on day 3 and RNA isolated for subsequent qPCR analysis. SPD-FL2siRNA treatment significantly reduced FL2 mRNA relative to SPD-Control-siRNA treatment. $n \geq 8$; nonparametric Mann-Whitney test; ${ }^{*} p=0.026$; error bars $=$ SEM. FL2, Fidgetin-like 2; mRNA, messenger RNA; qPCR, quantitative PCR; SEM, standard error of the mean; siRNA, small interfering RNA; SPD, surfactant polymer dressing. Color images are available online.

pared with their control counterpart (SPD with collagen microparticles containing nontargeting siRNA). These data indicate that FL2 siRNA was effectively being delivered and was causing degradation of FL2 mRNA transcripts at the site of application.

\section{SPD-FL2-siRNA expedites excisional wound re-epithelialization}

Following full-thickness excision, significant improvements in wound size were first noted in SPDFL2-siRNA-treated wounds 3 days post wounding
(Fig. 2A, B). On day 4, open wound areas of SPD alone and SPD-Control-siRNA-treated mice (21.8\% and $23.7 \%$ open, respectively) were nearly twice the size of SPD-FL2-siRNA-treated wounds (11.04\% open; $n=22$ wounds per group; Fig. 2C). Treatments continued through day 6 at which point wounds became too small to measure.

Histological analysis of wounds confirmed a significant reduction in wound size following SPDFL2-siRNA treatment; SPD-FL2-siRNA wounds were reduced to about half the length of controls
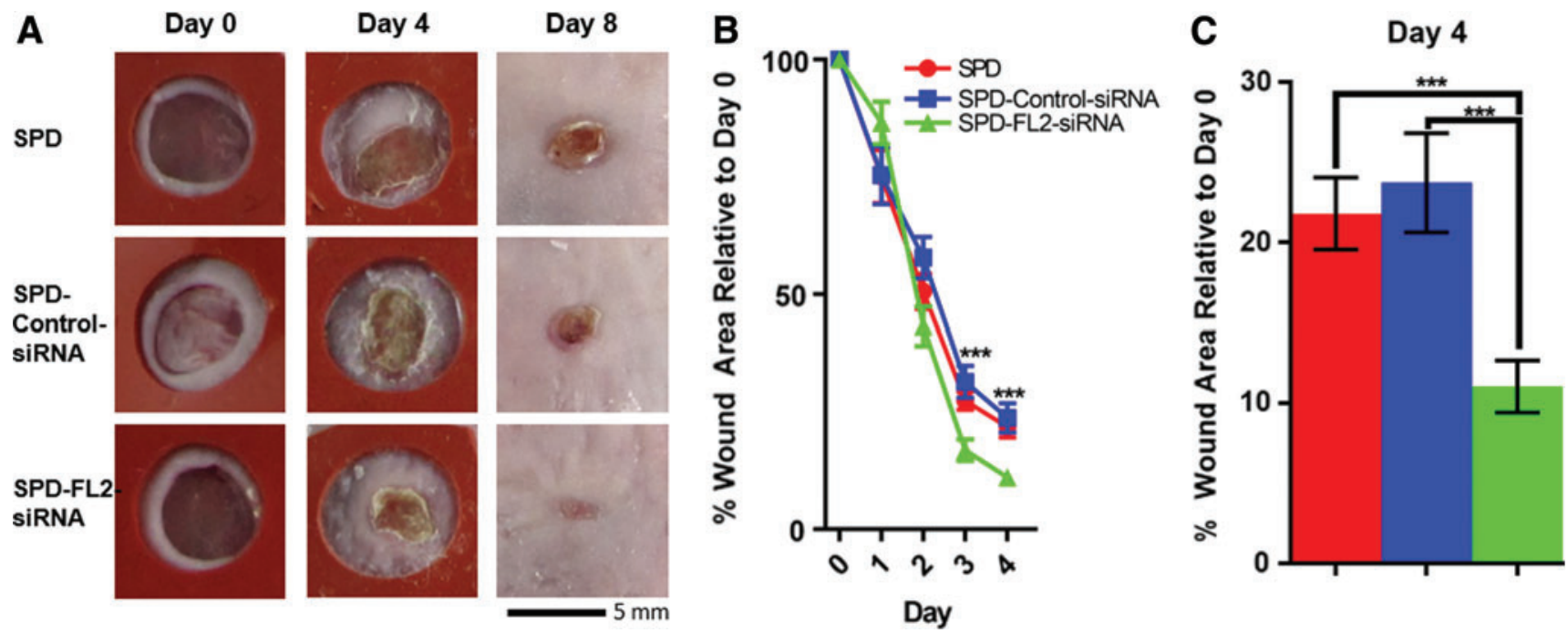

Figure 2. SPD-FL2-siRNA treatment stimulates healing in a mouse full-thickness excision model. (A) Representative time course of healing following the application of surfactant polymer treatment in BALB/c mice. (B, C) Graphs quantifying remaining wound area over time and at day 4 . Red bars are SPD treatment; blue bars, SPD-Control-siRNA; green bars, SPD-FL2-siRNA; $n=22$ wounds per group; Student's $t$-test; ${ }^{* * *} p \leq 0.0008$; error bars $=$ SEM. Significance confirmed at individual time points and with a two-way ANOVA. ANOVA, analysis of variance. Color images are available online. 


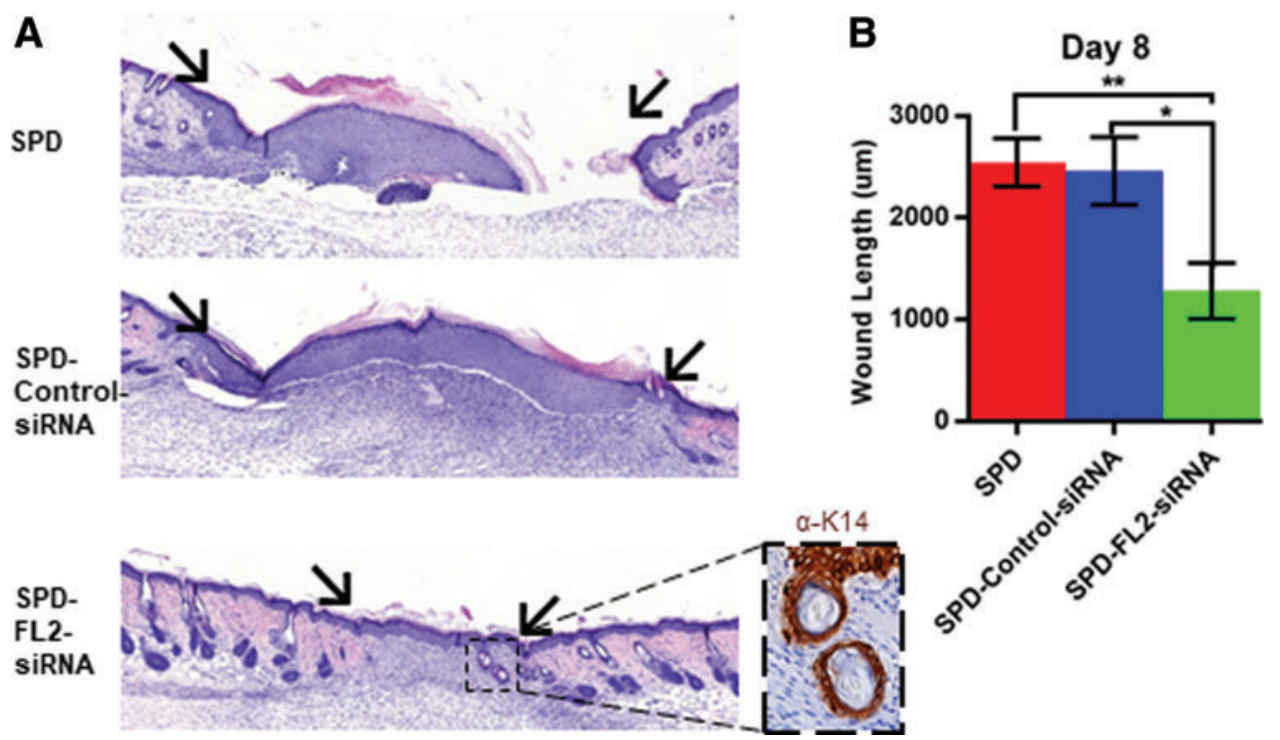

Figure 3. SPD-FL2-siRNA treatment reduces wound size in mouse full-thickness excision model. (A) Representative H\&E staining of bisected wounds taken 8 days after wounding. Epithelial thickening was present in the epidermis of the control groups, while FL2-siRNA-treated animals showed re-epithelialization, indicative of a further progressed, well-healing wound. Arrows designate observable wound areas. Inset image highlights keratin 14-positive hair follicles within the wound zone. (B) Graph quantifying wound length at day $8 ; n \geq 4$; Student's $t$-test; ${ }^{*} p=0.0252$; ${ }^{* *} p=0.0079$; error bars $=$ SEM. Color images are available online.

(average wound length of $2.5 \pm 0.2 \mathrm{~mm}$ for $\mathrm{SPD}$, $2.4 \pm 0.3 \mathrm{~mm}$ for SPD-Control-siRNA, and $1.2 \pm$ $0.3 \mathrm{~mm}$ for SPD-FL2-siRNA treatments, respectively; Fig. 3A, B). Furthermore, we noted that in a few cases ( 2 out of 7 ), SPD-FL2-siRNA-treated wounds had hair follicles present within the wound zone, as confirmed by keratin 14 staining (Fig. 3A inset). No such structures were observed in controls. These clinical and histological assessments indicate that SPD-FL2-siRNA was more effectively stimulating re-epithelialization and regeneration following full-thickness excision compared with controls.

\section{SPD-FL2-siRNA improves burn wound healing}

The effect of SPD-FL2-siRNA on wound healing was further tested in a full-thickness burn model. Burns were measured by two investigators blinded to the treatment groups using surgical calipers every day and tracked until closure (Fig. 4A, B). The wound sites enlarged in size for 4 days post injury, increasing up to $215 \%$ the size of the original burn area in control groups. SPD-FL2siRNA-treated burns, however, did not enlarge as dramatically, reaching a maximum of $160 \%$ the size of the original burn area. The reduced burn expansion was followed by significantly improved healing from day 4 to 9 compared with SPD and SPD-Control-siRNA-treated burns (Fig. 4B, C). Due to scabbing, burns became too difficult to measure using surgical calipers following day 9 .
Histological analysis of wound tissue 14 days post injury showed that SPD-FL2-siRNA-treated burns underwent a significant reduction in burn wound area (SPD and SPD-Control-siRNA were $35.5 \%$ and $39.3 \%$ larger, respectively; data not shown). All SPD-FL2-siRNA-treated burns had closed completely at day 14 , whereas $25 \%$ and $30 \%$ of SPD and SPD-Control-siRNA-treated wounds, respectively, remained unhealed (data not shown). Furthermore, SPD-FL2-siRNA-treated burns had a significant reduction in epidermal thickness compared with controls, more closely resembling normal skin (Fig. 4D). These clinical and histological assessments indicate that SPD-FL2siRNA promotes re-epithelialization and regeneration of wound tissue following full-thickness burns.

\section{SPD-FL2-siRNA improves regeneration of the skin}

We next characterized the quality of regenerated tissue through immunohistochemical staining.

First we looked to define FL2's role in cell proliferation as an increase in cell proliferation could potentially account for the shortened reepithelialization time. PCNA staining of burn wound sections revealed that there was no significant difference in the percentage of PCNApositive cells between the three treatment groups (Fig. 5A, B). These data indicate that FL2 knockdown does not affect cell proliferation during healing and suggests that cell migration is the 


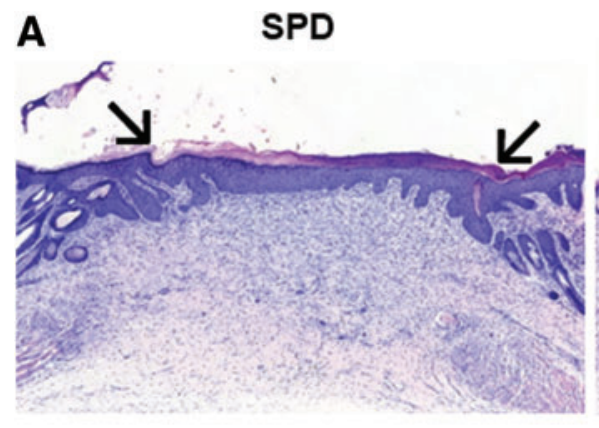

B

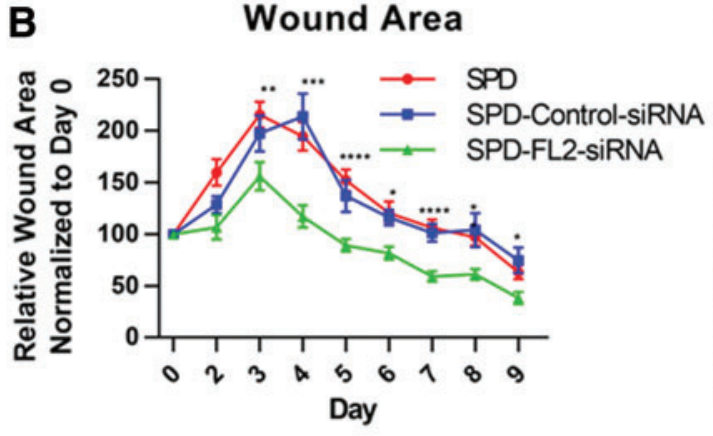

SPD-Control-siRNA

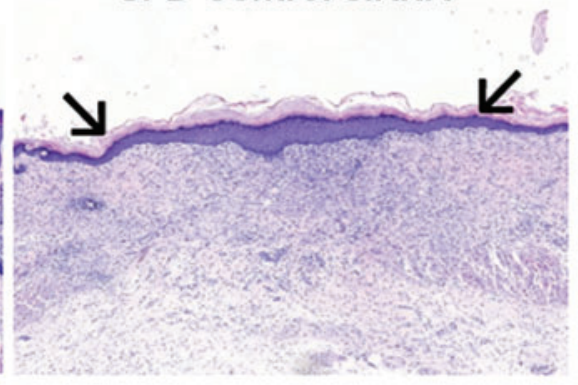

C

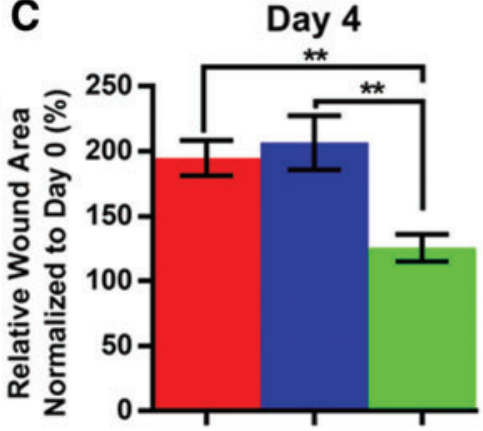

SPD-FL2-SiRNA

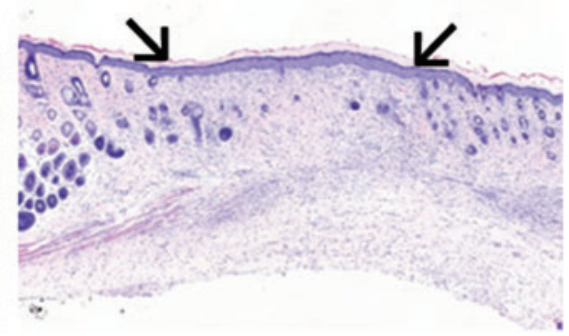

D Epidermal Thickness

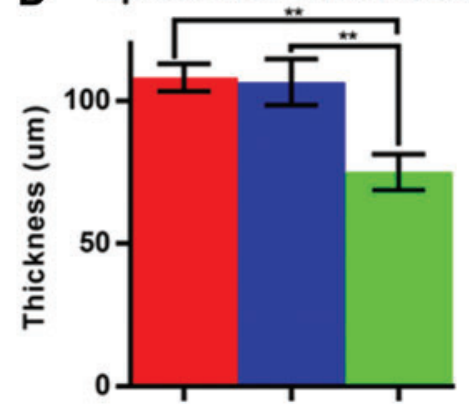

Figure 4. SPD-FL2-siRNA treatment stimulates healing in mouse full-thickness burn model. (A) Representative H\&E staining of bisected wounds taken 14 days post burning. FL2-siRNA-treated animals showed re-epithelialization, indicative of a further progressed, well-healing burn. Arrows designate observable wound edges. (B) Graph quantifying wound area over time relative to day 0 ; $n \geq 14$; significance confirmed at individual time points and with a twoway ANOVA. (C, D) Graphs quantifying wound area at day 4 and epidermal thickness at day 14. Red bars are SPD treatment; blue bars, SPD-Control-siRNA; green bars, SPD-FL2-siRNA; $n \geq 8$; Student's $t$-test; ${ }^{*} p \leq 0.05 ;{ }^{* *} p \leq 0.005 ;{ }^{* * *} p \leq 0.0005$; ${ }^{* * *} p \leq 0.00005$; error bars $=$ SEM. Color images are available online.

likely driver of hastened re-epithelialization compared with controls.

Managing inflammation is an essential process for effective wound healing. To assess the impact of FL2 knockdown on inflammatory cell presence at day 14, sections were stained for CD45, a marker of leukocytes and other immune cells. CD45 staining was present in all three treatment groups. However, we observed that SPD-FL2siRNA-treated wounds contained fewer distinct clusters of CD45-positive cells within the burn zone than SPD and SPD-Control-siRNA groups (Fig. 5A, C). After day 7, reduction of CD45positive cells was indicative of a return to baseline conditions. ${ }^{21}$

Revascularization of the burn zone was quantified by PECAM1 staining. Significantly more vessels per field were present in SPD-FL2-siRNAtreated wounds $(99.5 \pm 4.3$ vessels per field) than SPD and SPD-Control-siRNA treatments (73.5 \pm 6.8 and $62.7 \pm 7.9$ vessels per field, respectively; Fig. 5A, D). This data suggest that FL2 knockdown may enhance angiogenesis in wounded skin to provide oxygen and nutrients to the wound site for use in the rapidly regenerating wound zone.

\section{SPD-FL2-siRNA affects collagen III to collagen I ratios in burn scars}

In the early stages of wound healing, myofibroblasts deposit collagen III in the wound zone, increasing the ratio of collagen III to collagen I. Collagen III accounts for up to $50 \%$ of total collagen in the early stages of wound healing, up from levels in uninjured skin by about $20 \% .{ }^{24}$ As the scar matures, the ratio of collagen III to collagen I decreases to approximate normal levels.

To determine whether FL2 knockdown influences the ratio of collagen III to collagen I during burn healing, burns were analyzed using Herovici stains. Herovici is routinely used to quantify the ratio of collagen I (which stains red) and III (which stains blue) through multispectral imaging. Our results indicate that there were increased levels of mature collagen (reduction in collagen III to collagen I ratio) in SPD-FL2-siRNA-treated burn tissues, which further supports our hypothesis that FL2 knockdown accelerates wound healing.

\section{DISCUSSION}

Treating wounds with a SPD provides significant hydration to the wound site and increases 
A
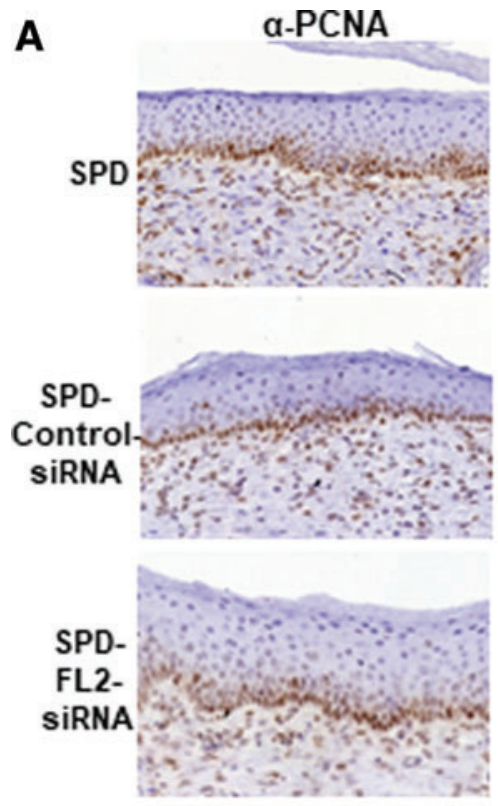

B

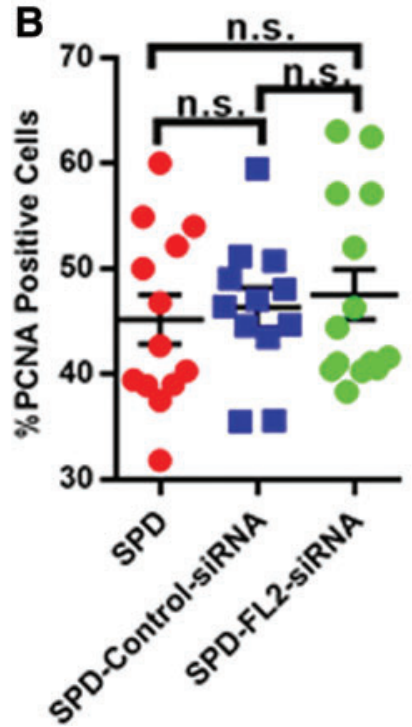

a-PECAM1
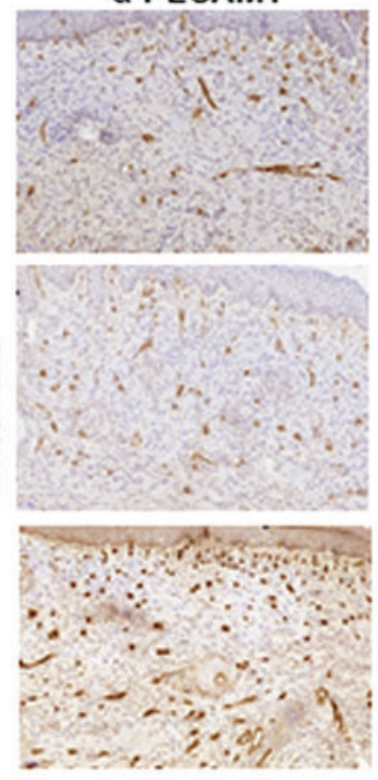

C

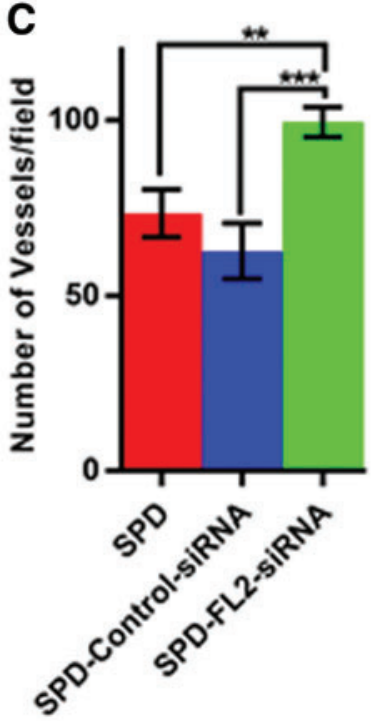

a-CD45
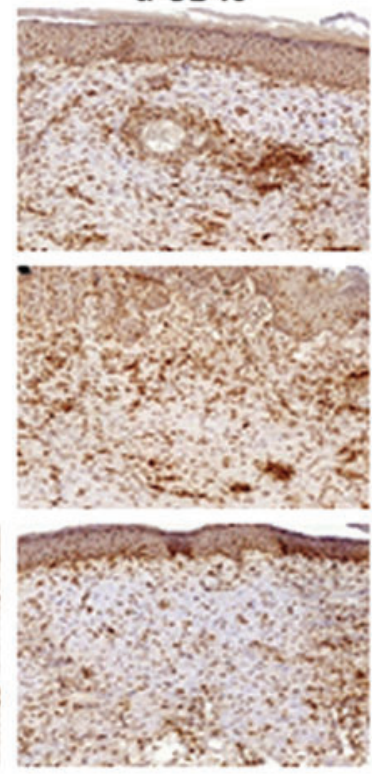

D

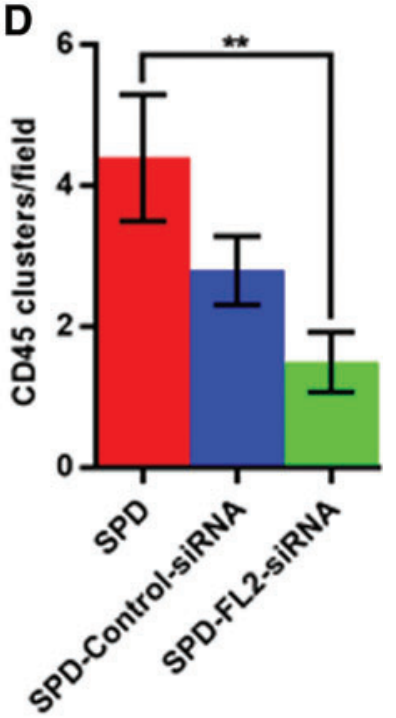

Figure 5. FL2 knockdown enhances revascularization and reduces inflammation without affecting cell proliferation. (A) Immunohistochemical staining of skin 14 days post burn and treatment using PCNA, PECAM1, or CD45 antibodies, respectively. Central regions of the burns were imaged at $20 \times$ or $40 \times$ magnification and the number of staining events quantified. (B) PCNA staining showed no significant differences between treatment groups; $n \geq 4$ wounds per condition, $\geq 3$ fields per wound; Student's $t$-test; n.s.=not significant. (C) Significant differences were observed between controls and SPD-FL2-siRNA treatment groups following PECAM1 staining; $n \geq 5$; Student's $t$-test; ${ }^{* *} p=0.0092$. (D) Significant differences were observed between controls and SPD-FL2-siRNA treatment groups following CD45 staining; $n \geq 10$; Student's $t$-test; $p={ }^{* *} 0.0027$; ${ }^{* * *} p=0.0003$; error bars $=$ SEM. CD45, cluster of differentiation 45 ; PCNA, proliferating cell nuclear antigen; PECAM1, platelet endothelial cell adhesion molecule-1. Color images are available online.

antimicrobial efficacy, improving healing outcomes. The data shown here support our hypothesis that the incorporation of FL2 siRNA, a therapeutic with a complimentary healing mechanism, into an SPD further improves wound-healing outcomes. Further development of alternative SPD formulations and optimization of dosing regimens is an area ripe for future investigation. $^{25}$

\section{Improving current therapeutic options with FL2-targeting siRNA}

Several wound-healing models, including dermal (excisional and burns), ocular (abrasions and burns), and $3 \mathrm{D}$ co-cultures of keratinocytes and fibroblasts, have underscored the importance of rapid reepithelialization as a key determinant of effective healing outcomes. ${ }^{26-32}$ The clinical and histological characterization of treated wounds presented 

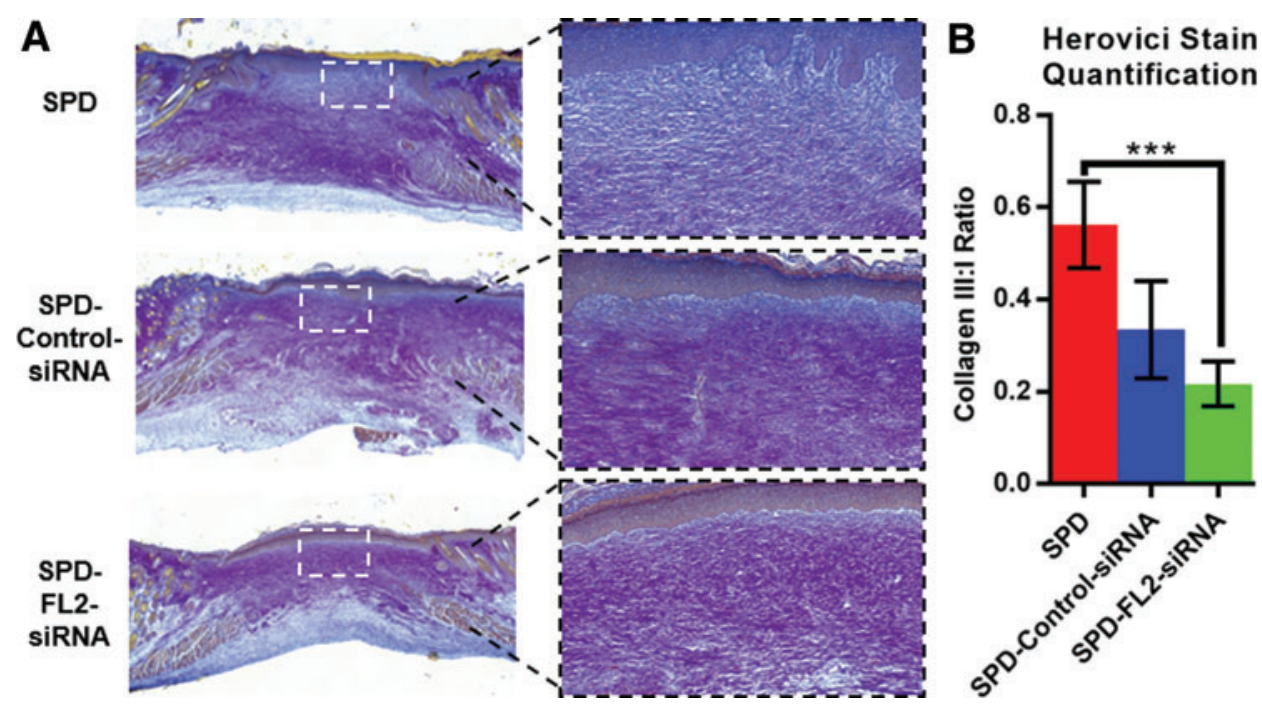

Figure 6. SPD-FL2-siRNA treatment enhances collagen remodeling in mouse full-thickness burn model. (A) Representative Herovici staining of bisected wounds taken 14 days post burning. FL2-siRNA-treated animals showed improved collagen maturation with a reduced collagen III (blue) to collagen I (red) ratio. Insets show $40 \times$ magnified view of wound zone. (B) Graph quantifying collagen ratio within the wound; $n \geq 11$; Student's $t$-test; ${ }^{* * *} p=0.001$; error bars $=$ SEM. Color images are available online.

above indicate that SPD-FL2-siRNA stimulates more efficient re-epithelialization and progresses wounds into subsequent stages of healing (resolution of inflammation) and regeneration (collagen maturation, angiogenesis) more quickly than controls.

The extent of inflammatory response following injury is a first indicator of potential wound outcome. ${ }^{33}$ It is noteworthy that SPD-FL2-siRNAtreated wounds show an expedited resolution of inflammation at earlier time points (Fig. 4B) and reduced $\mathrm{CD} 45^{+}$staining at later points (Fig. 5A); there is now evidence that more effective reepithelialization is correlated with shortened periods of inflammation and that reduced CD $45^{+}$levels 7 days post injury is indicative of further progressed wounds. ${ }^{21,34,35}$ Next, as re-epithelialization continues, leading edge cells generate a "healing" scaffold into the wound zone. This structure creates a path for cells to efficiently migrate to the center of the wound and begin remodeling the extracellular matrix. ${ }^{36}$ Our findings here show that FL2 knockdown influences collagen organization within the wound zone, reducing the ratio of collagen III to collagen I in treated wounds (Fig. 6). While increased deposition of collagen III is known to reduce the density of blood vessels within the wound, ${ }^{37}$ collagen I is an optimal substrate for endothelial cells, and this may explain the increase in angiogenesis seen in SPD-FL2siRNA-treated wounds (Fig. 5). ${ }^{38,39}$ Additional cell types such as epidermal stem cells also utilize this newly formed scaffold to migrate into the wound zone. ${ }^{36}$ We found that only FL2 siRNA-treated wounds showed stem cell-derived hair follicles within the wound zone, suggesting that these wounds were further progressed in the regeneration process (Fig. 3). Re-epithelialization also impacts local cellular signaling within the wound zone. Increasingly abundant keratinocytes release VEGF, recruiting endothelial cells into the wound zone, and may provide an alternative explanation for the increased angiogenesis seen in SPD-FL2siRNA-treated wounds. ${ }^{40}$ Additional studies will be needed to define these processes more completely.

In conclusion, we present a new SPD-siRNA platform that can be applied topically and used to treat excision wounds and burns. Treatment with SPD-FL2-siRNA depletes FL2 levels, which expedites re-epithelialization and ultimately improves dermal structure compared with treatment with SPD alone. Due to its favorable chemical composition and ease of application, an SPD-FL2-siRNA product could improve the lives of patients suffering from a variety of dermal injuries.

\section{INNOVATION}

Here we incorporated siRNA into SPD for use on cutaneous burns and excisions. To our knowledge this is the first time siRNA in collagen microparticles has been incorporated into SPD. While previous formulations of particles have been used to encapsulate siRNA, the platform presented herein has several advantages. SPD is biocompatible, 
nonionic, hydrating, and able to remain at the site of application. SPD also has noted anti-biofilm properties, helping to ward off infection and allowing wounds to heal. Combining SPD with the synergistic FL2 siRNA allows for the therapeutic use of both healing mechanisms.

\section{KEY FINDINGS}

- FL2 siRNA can be incorporated into current treatment options to further improve healing outcomes.

- The use of SPD improves the ease of application of FL2-siRNA.

- Collagen microparticles in SPD is a viable platform for testing alternative therapeutic siRNAs in wound-healing contexts.

\section{ACKNOWLEDGMENTS AND FUNDING SOURCES}

This work was supported in part by a grant from the Wound Healing Foundation. We would also like to acknowledge Einstein Analytical Imaging Facility (AIF) for expert advice/assistance in microscopy. The AIF is funded by the NCI Cancer Grant P30CA013330. Imaging was conducted using the 3DHistec Pannoramic 250 Flash II slide scanner, which was funded by the NIH 1S10OD019961-01. A.H.K. is partially supported by the National Research Foundation of South Africa.

\section{AUTHOR DISCLOSURE AND GHOSTWRITING}

B.O.R. is an employee of MicroCures, Inc. J.N. is a scientific advisor for MicroCures, Inc. D.J.S. is the founder and Chief Science Officer of MicroCures, Inc. D.J.S. is the owner of U.S. Patent \#20130022667 entitled "Fidgetin-like 2 as a target to enhance wound healing," which has been licensed by MicroCures. No other author has a conflict of interest. No ghostwriters were used to write this article.

\section{ABOUT THE AUTHORS}

David J. Sharp, PhD, is a Professor of Physiology and Biophysics at the Albert Einstein College of Medicine. He is the founder and Chief Science Officer of MicroCures, a company that develops therapeutics to enhance tissue regeneration and repair. Brian P. O'Rourke, PhD, is the lead scientist at MicroCures. Adam H. Kramer, MSc, is a graduate student in Dr. David J. Sharp's lab at the Albert Einstein College of Medicine. Longyue L. Cao, MD, PhD is a resident in the pediatrics program at Boston Children's Hospital. Mohammed Inayathullah, PhD, and Jayakumar Rajadas, PhD, run the Biomaterials and Advanced Drug Delivery lab (BioADD) at Stanford University. Hillary Guzik, MSc, is an imaging expert at the Analytical Imaging Facility at Albert Einstein College of Medicine. Josh D. Nosanchuck, MD, is Senior Associate Dean for Medical Education and Professor in the Departments of Medicine and Microbiology and Immunology at Albert Einstein College of Medicine. He also is a scientific advisor for MicroCures. Inc.

\section{REFERENCES}

1. Avishai E, Yeghiazaryan K, Golubnitschaja 0. Impaired wound healing: facts and hypotheses for multi-professional considerations in predictive, preventive and personalised medicine. EPMA J 2017;8:23-33

2. Raphael AP, Garrastazu G, Sonvico F, Prow TW. Formulation design for topical drug and nanoparticle treatment of skin disease. Ther Deliv 2015;6:197-216

3. Werner S, Grose R. Regulation of wound healing by growth factors and cytokines. Physiol Rev 2003;83:835-870.

4. Liang $H$, Nacharaju $P$, Friedman A, Friedman JM. Nitric oxide generating/releasing materials. Future Sci OA 2015;1. DOI: 10.4155/fso .15.54.

5. Greenhalgh DG. The role of growth factors in wound healing. J Trauma 1996;41:159-167.
6. Ahmed EM. Hydrogel: preparation, characterization, and applications: a review. J Adv Res 2015; 6:105-121.

7. Das Ghatak P, Mathew-Steiner SS, Pandey P, Roy S, Sen CK. A surfactant polymer dressing potentiates antimicrobial efficacy in biofilm disruption. Sci Rep 2018;8:873.

8. Blache U, Ehrbar M. Inspired by nature: hydrogels as versatile tools for vascular engineering. Adv Wound Care 2018;7:232-246.

9. Greenebaum B, Blossfield K, Hannig J, et al. Poloxamer 188 prevents acute necrosis of adult skeletal muscle cells following high-dose irradiation. Burns 2004;30:539-547.

10. Merchant FA, Holmes WH, Capelli-Schellpfeffer M, Lee RC, Toner M. Poloxamer 188 enhances functional recovery of lethally heat-shocked fibroblasts. J Surg Res 1998;74:131-140.
11. de Araújo DR, Oshiro A, da Silva DC, Akkari ACS, de Mello JC, Rodrigues T. Alessandra Cristina Santos Akkari, Joyce Cristine de Mello, Tiago Rodrigues, Poloxamers as Drug-Delivery Systems: physicochemical, London, UK: Pharmaceutical, and Toxicological Aspects, 2013

12. Charafeddine RA, Makdisi J, Schairer D, et al. Fidgetinlike 2: a microtubule-based regulator of wound healing. J Invest Dermatol 2015;135:2309-2318.

13. Hughes OB, Rakosi A, Macquhae F, Herskovitz I, Fox JD, Kirsner RS. A review of cellular and acellular matrix products: indications, techniques, and outcomes. Plast Reconstr Surg 2016;138:138s-147s.

14. PluroGen, What is PluroGel. http://plurogen.com/ what-is-plurogel/ (last accessed October 16, 2018).

15. ClinicalTrials.gov, PluroGel on Wounds of Mixed Etiology. https://clinicaltrials.gov/ct2/show/ NCT03275831 (last accessed October 16, 2018). 
16. Palumbo FP, Harding KG, Abbritti F, et al. New surfactant-based dressing product to improve wound closure rates of nonhealing wounds: a European multicenter study including 1036 patients. Wounds 2016;28:233-240.

17. Glim JE, van Egmond M, Niessen FB, Everts V, Beelen RH. Detrimental dermal wound healing: what can we learn from the oral mucosa?, Wound Repair Regen 2013;21:648-660.

18. Dunn L, Prosser HC, Tan JT, Vanags LZ, Ng MK, Bursill CA. Murine model of wound healing. J Vis Exp 2013;75:e50265. DOI: 10.3791/50265.

19. Cai EZ, Ang CH, Raju A, et al. Creation of consistent burn wounds: a rat model, Arch Plast Surg 2014;41:317-324.

20. Jeschke MG, Barrow RE, Hawkins HK, Tao Z, Perez-Polo JR, Herndon DN. Biodistribution and feasibility of non-viral IGF-I gene transfers in thermally injured skin. Lab Invest 2000;80:151-158.

21. Suga $H$, Rennert RC, Rodrigues $M$, et al. Tracking the elusive fibrocyte: identification and characterization of collagen producing hematopoietic lineage cells during murine wound healing. Stem Cells 2014;32:1347-1360.

22. Turner NJ, Pezzone MA, Brown BN, Badylak SF. Quantitative multispectral imaging of Herovici's polychrome for the assessment of collagen content and tissue remodelling. J Tissue Eng Regen Med 2013;7:139-148.

23. Rawlins JM, Lam WL, Karoo RO, Naylor IL, Sharpe DT. Quantifying collagen type in mature burn scars: a novel approach using histology and digital image analysis. J Burn Care Res 2006;27:60-65.

24. Xue M, Jackson CJ. Extracellular matrix reorganization during wound healing and its impact on abnormal scarring. Adv Wound Care 2015;4:119136.

25. Wang LL, Burdick JA. Engineered hydrogels for local and sustained delivery of RNA-interference therapies. Adv Healthc Mater 2017. [Epub ahead of print]; DOI: 10.1002/adhm.201601041.

26. Larjava H, Koivisto L, Häkkinen L. Keratinocyte Interactions with Fibronectin during Wound Healing. Landes Bioscience, Madame Curie Bioscience Database [Internet]. Austin, TX: Landes Biosciences, 2000-2013.

27. Wierzbicka-Patynowski I, Schwarzbauer JE. The ins and outs of fibronectin matrix assembly. J Cell Sci 2003;116:3269-3276.

28. Yates CC, Hebda P, Wells A. Skin wound healing and scarring: fetal wounds and regenerative restitution. Birth Defects Res C Embryo Today 2012 96:325-333.

29. Spyrou GE, Watt DA, Naylor IL. The origin and mode of fibroblast migration and proliferation in granulation tissue. Br J Plast Surg 1998;51:455-461.

30. Sorrell JM, Baber MA, Caplan Al. Site-matched papillary and reticular human dermal fibroblasts differ in their release of specific growth factors/ cytokines and in their interaction with keratinocytes. J Cell Physiol 2004;200:134-145.

31. Beyeler J, Schnyder I, Katsaros C, Chiquet M. Accelerated wound closure in vitro by fibroblasts from a subgroup of cleft lip/palate patients: role of transforming growth factor-alpha. PLoS One 2014;9:e111752

32. Varkey M, Ding J, Tredget EE. Fibrotic remodeling of tissue-engineered skin with deep dermal fibroblasts is reduced by keratinocytes. Tissue Eng Part A 2014;20:716-727.

33. DiPietro LA. Angiogenesis and wound repair: when enough is enough. J Leukoc Biol 2016;100: 979-984.

34. Li D, Wang A, Liu X, et al. MicroRNA-132 enhances transition from inflammation to proliferation during wound healing. J Clin Invest 2015;125:3008-3026.

35. Junker JPE, Kamel RA, Caterson EJ, Eriksson E. Clinical impact upon wound healing and inflam- mation in moist, wet, and dry environments. Adv Wound Care 2013:2·348-356.

36. Aragona M, Dekoninck S, Rulands S, et al. Defining stem cell dynamics and migration during wound healing in mouse skin epidermis. Nat Commun 2017;8:14684

37. Tonnesen MG, Feng $X$, Clark RAF. Angiogenesis in wound healing. J Investig Dermatol Symp Proc 2000;5:40-46.

38. Davis GE, Senger DR. Endothelial extracellular matrix: biosynthesis, remodeling, and functions during vascular morphogenesis and neovessel stabilization. Circ Res 2005:97:1093-1107.

39. Twardowski T, Fertala A, Orgel JP, San Antonio JD. Type I collagen and collagen mimetics as angiogenesis promoting superpolymers. Curr Pharm Des 2007;13:3608-3621.

40. Frank S, Hubner G, Breier G, Longaker MT, Greenhalgh DG, Werner S. Regulation of vascular endothelial growth factor expression in cultured keratinocytes. Implications for normal and impaired wound healing. J Biol Chem 1995;270: 12607-12613.

$$
\begin{aligned}
& \text { Abbreviations and Acronyms } \\
& \text { ANOVA }=\text { analysis of variance } \\
& \text { CD45 }=\text { cluster of differentiation } 45 \\
& \text { FL2 }=\text { Fidgetin-like } 2 \\
& \text { mRNA }=\text { messenger RNA } \\
& M T=\text { microtubule } \\
& \text { PBS }=\text { phosphate buffered saline } \\
& \text { PCNA }=\text { proliferating cell nuclear antigen } \\
& \text { PECAM1 }=\text { platelet endothelial cell } \\
& \text { qPCR }=\text { adhesion molecule-1 } \\
& \text { SEM }=\text { standative PCR } \\
& \text { SiRNA }=\text { small interfer of the mean } \\
& \text { SPD }=\text { surfactant polymer dressing }
\end{aligned}
$$

